University of Tennessee Health Science Center

UTHSC Digital Commons

Fall 10-17-2021

\title{
The Impact of the Health Information Technology for Economic and Clinical Health Act on Clinical Pharmacy and Computerized Provider Order Entry
}

Bailey Deglow

University of Tennessee Health Science Center

Follow this and additional works at: https://dc.uthsc.edu/hiimappliedresearch

Part of the Health and Medical Administration Commons, and the Health Information Technology Commons

\section{Recommended Citation}

Deglow, Bailey, "The Impact of the Health Information Technology for Economic and Clinical Health Act on Clinical Pharmacy and Computerized Provider Order Entry" (2021). Applied Research Projects. 78. .

https://doi.org/10.21007/chp.hiim.0075

https://dc.uthsc.edu/hiimappliedresearch/78

This Research Project is brought to you for free and open access by the Department of Health Informatics and Information Management at UTHSC Digital Commons. It has been accepted for inclusion in Applied Research Projects by an authorized administrator of UTHSC Digital Commons. For more information, please contact jwelch30@uthsc.edu. 
The Impact of the Health Information Technology for Economic and Clinical Health Act on Clinical

Pharmacy and Computerized Provider Order Entry

Bailey Deglow, PharmD, MHIIM Candidate

The University of Tennessee Health Science Center

College of Health Professions

Advisor: Sajeesh Kumar, PhD

Fall 2021 


\section{Acknowledgement}

I would first like to thank my advisor, Dr.Sajeesh Kumar, for helping me with this research project. We have met every few weeks for over six months, and I could not have finished this project without him. I would also like to thank Dr. Meagan Phelps for all of her assistance with this research. She has helped me tremendously and encouraged me to become a better pharmacist. Next, I would like to thank my roommate, Zoe, for listening to me when I was overwhelmed about the paper and giving me advice about life in general. Finally, I would like to thank my parents for being my biggest cheerleaders and helping me to pursue the MHIIM degree. I would not be graduating without them. 


\begin{abstract}
The Health Information Technology for Clinical and Economic Health (HITECH) Act of 2009 encouraged the meaningful use of the electronic health record (EHR) by health care providers in the United States. "Meaningful use" monetary incentives were offered by the Center for Medicare and Medicaid Services (CMS) for health care providers who met standards of documenting in and using the EHR. While clinical pharmacists typically work in clinics and hospitals in the United States, they were not considered eligible professionals who could receive incentives for using the EHR. There is a great deal of literature regarding the use of the EHR by eligible professionals, but not by ineligible professionals like clinical pharmacists. One way that clinical pharmacists assist in meaningful use criteria is by developing computerized provider order entries (CPOEs). The purpose of this study is to assess the perception and use of CPOEs by clinical pharmacists.
\end{abstract}




\section{Chapter 1}

\section{Introduction}

In response to proof that paper-based records would increase health care costs, fragment care, and cause medical decisions to be made without complete data, Congress signed the Health Information Technology for Economic and Clinical Health (HITECH) Act into law on February 17, 2009. HITECH was part of the American Recovery and Reinvestment Act and had a goal of increasing the adoption of the electronic health record (EHR). In fact, HITECH mandated the adoption of the EHR and incentivized health care providers to promote the "meaningful use" of it (HITECH, 2009). This meant that not only did health care providers need to learn about and begin documenting patient information into the EHR, but they also needed to use it in a way that would benefit the US health care system to ensure patients received quality, timely, and affordable health care. Prior to the HITECH Act, many health care providers were primarily using paper-based forms of health information documentation (Anderegg \& Gumpper, 2012). With the adoption of meaningfully using the EHR, it offered providers a way to optimize qualitybased care. It also allowed an interdisciplinary approach to care because the EHR would allow viewing from multiple providers at the same time. This opened the door for pharmacists, specifically clinical pharmacists, to have a greater impact on patient care.

The payment incentives for meeting the meaningful use criteria were provided yearly, beginning in 2011, by the Center for Medicare and Medicaid Services (CMS) to "eligible professionals" and "eligible hospitals." Table 1 displays the eligible professionals and hospitals that could receive the incentive payments. Each eligible category has its own stipulations in regard to practice setting, and whether it's part of Medicare, Medicaid, or both. Pharmacists were not classified as eligible professionals, while physicians, nurse practitioners, dentists, etc. were. Because of this, to assist in meeting criteria for meaningful use, clinical pharmacists were the type of pharmacists that would greatly be able to assist in obtaining meaningful use criteria because they can practice in eligible hospitals. The American College 
of Clinical Pharmacy (ACCP) defines a clinical pharmacist as a pharmacist that "works directly with physicians or other health professionals and patients to ensure that the medications prescribed for patients contribute to the best possible outcomes," (ACCP, 2020). Clinical pharmacists practice in acute care hospitals, ambulatory care clinics, and diabetes and anticoagulation clinics to help manage patients' medication interactions, dosing, duration, etc. Clinical pharmacists are part of the interdisciplinary team to help manage patient care in each of those settings.

Table 1. Eligible Professionals and Hospitals for CMS Meaningful Use Incentive Payments

\begin{tabular}{|l|c|c|}
\hline Eligible Hospitals & Medicare Incentive Program & Medicaid Incentive Program \\
\hline Acute Care Hospitals & $\checkmark$ & $\checkmark$ \\
\hline Critical Access Hospitals & $\checkmark$ & $\checkmark$ \\
\hline Indian Health Service Hospitals & $\checkmark$ & $\checkmark$ \\
\hline Children's Hospitals & & $\checkmark$ \\
\hline Cancer Hospitals & \multicolumn{1}{|c|}{} \\
\hline Territory Hospitals & Medicare Incentive Program & Medicaid Incentive Program \\
\hline Eligible Professionals & $\checkmark$ & $\checkmark$ \\
\hline Physicians (MD/DO) & $\checkmark$ & $\checkmark$ \\
\hline Podiatrists (DPM) & $\checkmark$ & \\
\hline Optometrists (OD) & $\checkmark$ & $\checkmark$ \\
\hline Chiropractors (DC) & & $\checkmark$ \\
\hline Nurse Practitioners & $\checkmark$ & $\checkmark$ \\
\hline Dentists/Oral Surgeons (DDS/DMD) & & $\checkmark$ \\
\hline Physician Assistants (PA-C) & & \\
\hline Certified Nurse Midwives (CNM) & \multicolumn{1}{|c|}{} \\
\hline
\end{tabular}

Mennemeyer et al. (2017) explained, "By the end of 2014, the federal government had distributed $\$ 28.1$ billion to eligible physicians and other professionals through the Medicare and Medicaid EHR MU programs," (p.375). The meaningful use criteria were categorized into three stages. Stage 1 criteria were released in 2011 and focused on setting the foundational goals for using the EHR. Stage 1 included objectives such as: maintaining a problem list and active medication and allergy list, recording patient demographics, smoking status, clinical summary education, and medication reconciliation. Stage 2 criteria were released in 2014 and advanced clinical processes which included: 
inputting vital signs, providing timely access to patient health information, transmitting patients' health information to a third party, providing reminders for follow-up and educational resources, and recording family history, etc., (Alfayez, 2018). Stage 3, the most recently released stage, contained measures that aimed to promote HER interoperability. Protecting electronic protected health information, generating electronic prescriptions, using computerized provider order entry (CPOE) for medication or laboratory orders, and implementing clinical decision support systems (CDSs) were some of the objectives outlined in the stage 3 (CMS, 2018). For eligible hospitals, there were 6 objectives that that needed to be met. In November of 2016, CMS released a final rule that modified criteria due to response of stakeholder feedback.

While clinical pharmacists were not "eligible professionals" who received monetary incentives from the Centers for Medicare and Medicaid Services (CMS) for meaningful use, the profession had to adapt to using the EHR in eligible hospitals, whether that be by documenting care objectives into patient notes or creating medication-based templates for computerized provider order entry (CPOE). CPOE "refers to the process of providers entering and sending treatment instructions - including medication, laboratory, and radiology orders - via computer application (ONC, 2018). With pharmacists' extensive knowledge regarding drug dosing, interactions, adverse reactions, and effectiveness, they are important professionals when it comes to optimizing medication orders in hopsitals. In fact, one of the clinical pharmacy roles that evolved because of HITECH and CMS Meaningful Use was helping to create medication CPOE templates to improve patient care. Prgomet et al. (2017) completed a meta-analysis looking at the effect of CPOE and CDSs on medication errors, length of stay, and mortality in intensive care units. Their analysis results showed that when compared to paper-based ordering, "CPOE systems in intensive care units were associated with $85 \%$ reduction in medication prescribing error rates and $12 \%$ reduction in intensive care unit mortality rates (p. 415). 
Ascension Saint Thomas Rutherford Hospital (ASTR), which qualifies as an acute care hospital in Murfreesboro, TN, has a committee made up of physicians, pharmacists, nurses, and other key stakeholders that work together to create CPOEs called Powerplans. Clinical pharmacists play a major role in helping to format the medication CPOE templates. The medication CPOE templates are created for a number of reasons.

\section{The Purpose and Significance of the Study}

While there are a great deal of scholarly articles that have been published regarding the use and the adoption of the EHR by eligible professionals, there is a lack of data regarding its use by ineligible professionals. Pharmacists, and specifically clinical pharmacists in eligible hospitals, have been incorporated into the interdisciplinary team and are a valued healthcare professional in the hospital setting. The purpose of this study is to understand the impact that pharmacists believe medication CPOEs have had on patient outcomes (reduced medication errors (ADEs), adverse drug reactions, length of stay, mortality, correct dosing of drugs, antimicrobial stewardship, drug interactions) at ASTR and to describe the new role that clinical pharmacists have to develop these CPOEs because of HITECH and CMS meaningful use. 


\section{Chapter 2}

\section{Literature Review}

\section{Method}

The literature articles addressed the adoption of HITECH and meaningful use by hospitals and health care providers. The articles were found by searching PubMed. 2010 was the earliest year that articles were shown. The search terms included: HITECH, HITECH Act, pharmacy, meaningful use, EHR, and CPOE. Key concepts from one article provided information relevant for an overview of HITECH and the future of it. The article by Washington, DeSalvo, and Mostashari discussed the adoption of EHR as reported by physicians at their practice sites. The article explains that "Today, almost all U.S. hospitals and nearly $80 \%$ of office-based practices use certified EHRs" (2017). They provided a graph (see Figure 1) that displays a timeline of adoption of "any", "basic", and "certified" EHR in both office-based and acute care hospital settings. 


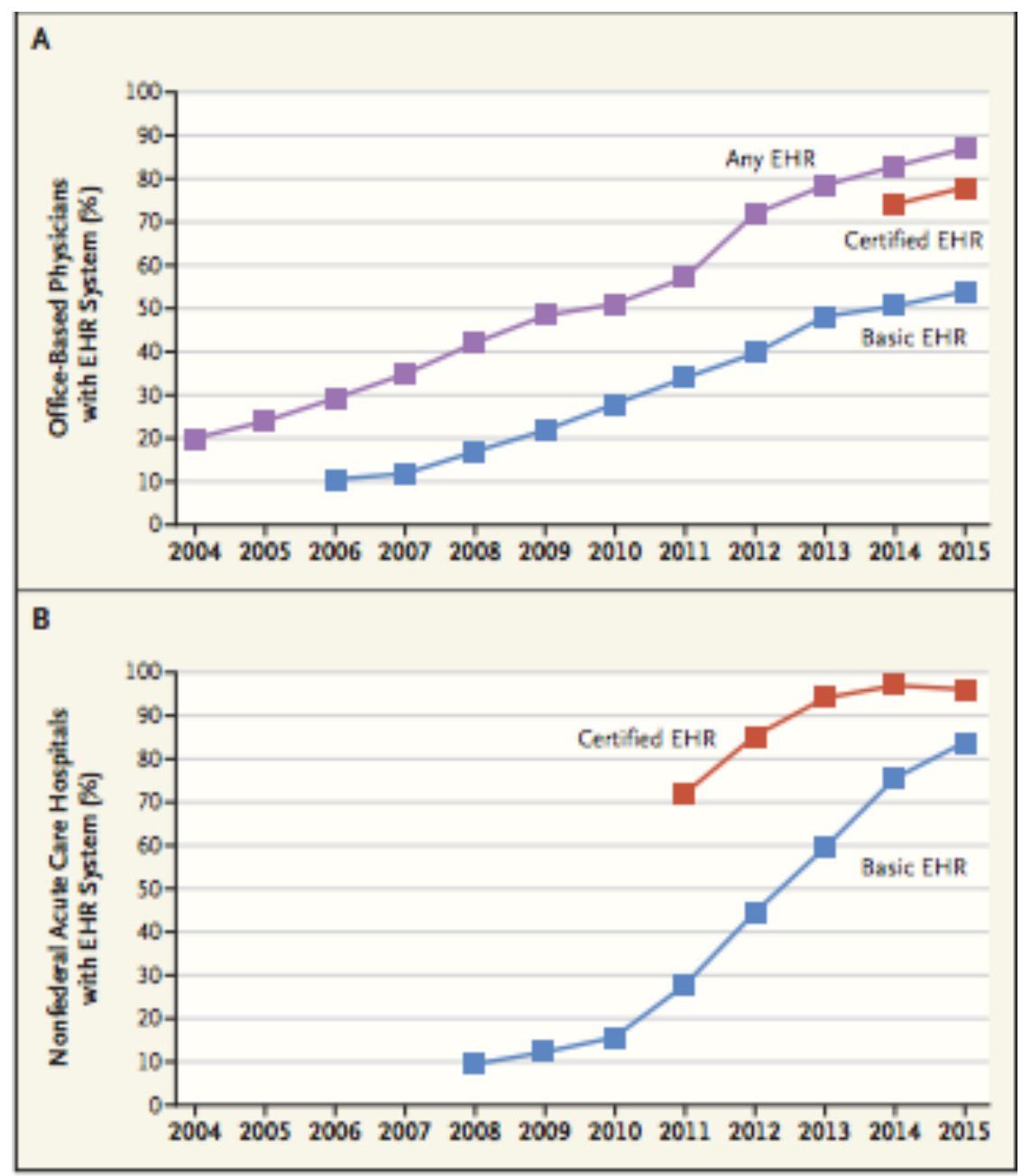

Figure 1 (Washington et al.)

Any EHR means that physicians have reported that their location used an electronic health record. Basic EHR means the location documented every computerized function (patient demographics, imaging results, patient medications, allergies, etc.) into the EHR. Certified EHR indicates that a physician reported that their location's EHR met the criteria for meaningful use. This is important to note because while hospitals had "Basic EHR" reported in 2008, they did not meet meaningful use criteria until 2011. A similar timeline is reported in office-based practices as well.

"Health care providers, especially physicians, have borne the brunt of this transformation," (Washington et al., 2017). This article primarily surveyed physicians, it did not survey pharmacists. Another article in this literature review discusses the major issues that pharmacists have had when 
documenting into the EHR. Other concepts in this article are important to include in this literature review like the fact that regulation is still an obstacle with health information technology and the limitations with billing and compliance needs.

\section{Populations Studied}

The second article goes into more detail and discusses pharmacists and their role in documenting meaningful use objectives into the EHR, specifically stages 1 and 2 . Alfayez et al. states, "Stage 1 concentrates on capturing and sharing data, Stage 2 on advanced care processes and integration into patient care, and Stage 3 on improving patient outcomes," (2018). One of the main points of the article is that pharmacists are not considered "eligible professionals" that can receive payment incentives for reaching objectives. This article studies El Rio Community Health Center which is health care system that provides care to medically underserved in Arizona. For approximately six inconsecutive months, clinical pharmacists' and other health care providers' documentation was tracked using their health information system. The specific patient population that was studied was patients with diabetes mellitus.

\section{Study Method}

The literature review had several different types of surveying done in each article. The first articlce by Washington et al. used surveying of physicians to report their adoption level of EHR (2017). The second article by Alfayez et al. was a "retrospective observational study of randomly selected patients with type 2 diabetes," (2018). Approximately 800 encounters were tracked for MU objectives using the NextGen EHR. The final study looked at pharmacists' role in documentation and medication reconciliation in a the EHR.

\section{Findings}

From reviewing the literature, HITECH of 2009 has achieved its intended purpose to expand the adoption of EHR and expand the economy in the US. In 2017, Washington et al. stated that "almost all 
US hospitals and nearly $80 \%$ of office-based practices use certified EHRs (2017). "Certified EHR" meant that not only had the practice site used an EHR, but it also used it in a way that met criteria of meaningful use. The payment incentives for meeting meaningful use criteria were provided yearly by the Center for Medicare and Medicaid Services and pharmacists were not classified as "eligible professionals" to receive the incentive payments. The meaningful use criteria were broken up into three stages. Stage 1 criteria included the following: maintaining a problem list, maintaining an active medication and allergy list, recording patient demographics, smoking status, clinical summary education, and medication reconciliation. Stage 2 criteria became more specific and included stage 1 criteria plus: vital signs, providing timely online access to health information, transmitting patients' health information to a third party, providing reminders for follow-up and educational resources, and recording family history (Alfayez et al., 2017). Stage 3 objectives included: implementing clinical decision support interventions (CDS), using computerized provider order entry (CPOE), public health reporting, providing patients with timely electronic access to health information, and having protected health information, (CMS, 2020).

The American College of Clinical Pharmacy defines a clinical pharmacist as a pharmacist that "works directly with physicians other health professionals, and patients to ensure that the medications prescribed for patients contribute to the best possible health outcomes." Clinical pharmacists are located in acute care hospitals, ambulatory care clinics, and diabetes and anticoagulation clinics to help manage patients' medication interactions, dosing, duration, etc. In the clinical setting, pharmacists use the EHR to document and update medication lists, complete medication reconciliations, patient evaluation and drug monitoring (Nelson et al., 2017). The expanded role of clinical pharmacists contributes to documenting in the EHR on a regular basis in many hospitals. And in the study by Alfayez et al., it was concluded that clinical pharmacists were "at least as effective as other health care providers in documenting meaningful use criteria, (2017). Pharmacists can also create templates for optimally 
dosing medicine. This is called clinical decision support tools, and these tools ease the providers prescribing if they are created in a manner that is easy to read and follow. However clinical decision support by clinical pharmacists remains underutilized (Nelson et al.) Pharmacists are not "eligible professionals", yet from the literature, they contribute to the EHR in numerous ways. As telehealth emerges as a way to provide care to patients without being in person with a provider, it is equally, if not more important, to use the EHR more efficiently and effectively with a pharmacist's knowledge and support.

\section{Limitations}

Limitations to the Washington et al. are the persons reporting. The study surveyed only physicians and their attestations to using EHR. The study could be a better representation of use if it included reports from nurses, nurse practitioners, PAs, and other eligible professionals. Some of the limitations to the Alfayez et al. study are the locations. The study was completed in a health system for the underserved in Arizone. Also, the patient population was patients with type 2 diabetes mellitus. To broaden the concept, it would be neat to see another health system and a different patient population for the encounters to occur. The study also solely focused on stage 1 and stage 2 of meaningful use, stage 3 documentation would broaden the scope of pharmacist documentation and provide a more recent study.

\section{Conclusions}

There are still many implications to improve the use of EHR in health systems as well as many regulatory challenges. Pharmacists play a role in updating medication lists in patient profiles, documenting patient allergies, providing CPOE, CDS, and in El Rio Community Health Center, pharmacists met some meaningful use objectives more than other providers. HITECH impacted health systems across the US and initiated the creation of health information system companies as well as provided a commonplace to document patient health information. The EHR allows information to be 
used with an interdisciplinary approach and pharmacists have incorporated themselves into the process to optimize patient care. 


\section{Chapter 3}

\section{Purpose}

The purpose and primary endpoint of this study is to determine the perception and use of CPOEs by clinical pharmacist. Secondary endpoints include clinical pharmacists' perspective of PowerPlans (their term for CPOEs within their health system) impact on medication safety, antimicrobial stewardship, and involvement in the creation of their PowerPlans.

\section{Research Questions}

How to clinical pharmacists describe their knowledge of CPOE and its use? Do pharmacists at Ascension Saint Thomas Rutherford Hospital believe that Powerplans help to prevent medication errors, morality, and length of stay? Do they recommend the use of PowerPlans in institutional and clinical settings throughout the United States?

\section{Methods}

\section{Participants and Research Site}

The participants of this survey included all pharmacists at Ascension Saint Thomas

Rutherford (ASTR), Murfreesboro, TN, USA. ASTR is a 358-bed hospital and averages 12,000 admissions and 90,000 emergency room visits annually. Services at ASTR include heart and vascular care, orthopedics, intensive care, and maternity services. ASTR is member of Saint Thomas Health and is the leading health care provider in Rutherford County just outside of Nashville. Rutherford County has a population of nearly 288,906 in 2014, an increase of more than 50 percent since 2000 (Ascension, 2021).

There are approximately 30 clinical pharmacists on staff at ASTR. The pharmacy model at ASTR is decentralized, meaning the pharmacists are not working solely in the hospital pharmacy, but practicing in other areas throughout the hospital with the nurses and doctors to answer any drug-related questions and giving suggestions to optimize patient care. They work in many areas of the hospital 
including: emergency department, critical care units, medical/surgery, and on the in-patient floors. Participants received informed consent.

The health information system used at ASTR is Cerner. Within Cerner, pharmacists, nurses, physicians, and other stakeholders create order sets, called PowerPlans within the Cerner EHR. These PowerPlans are a form of CPOE and allow for simultaneous ordering of all components associated with clinical care and drug monitoring. This includes lab tests, $x$-rays, and medications. One example of how a PowerPlan can be used is when a health care provider is attempting to order oral vancomycin to treat a Clostridioides difficile infection, the order will prompt the provider to discontinue all medications that have been shown to cause a Clostridioides difficile infection. Without that prompt, a provider may forget to discontinue those medications and the patient would remain on them while trying to treat the infection that they thought to be causing. Another type of CPOE that is created is one for a thrombolytic that is used to treat an ischemic stroke. Thrombolytics are very effective in dissolving the clot, but they have many contraindications to their use that includes a time limit for their use. Clinical pharmacists are aware of these contraindications, and therefore can greatly assist in the creation of the CPOE template for the thrombolytics. These CPOEs would help prevent the use of thrombolytics when they are contraindicated and therefore increase medication safety.

\section{Research Design and Data Collection}

In this single center, cross sectional study, practicing pharmacists at ASTR were emailed a link to a Google Forms survey. They had approximately 14 days to complete and submit the survey. The survey questions were multiple choice with one free text question. There were seventeen total questions, four of them were demographic-based questions to better gauge the pharmacy population, and thirteen of them were Likert scale to assess the information related to the purpose of the study. Written informed consent was given to each of the participants. IRB approval was not required. A copy of the questions in the survey are included below. 
1. How do you describe your knowledge of computerized provider order entry (CPOE) and its use?

- Extremely knowledgeable

- Very knowledgeable

- Moderately knowledgeable

- Slightly knowledgeable

- Not at all knowledgeable

2. How effective are PowerPlans in reducing medication errors at ASTR?

- Extremely effective

- Very effective

- Moderately effective

- Slightly effective

- Not at all effective

3. How helpful are PowerPlans to improve clinical decisions at ASTR?

- Extremely helpful

- Very helpful

- Moderately helpful

- Slightly helpful

- Not at all helpful

4. To what extent do PowerPlans increase antimicrobial stewardship at ASTR?

- Extremely high

- Very high

- Moderately high

- Slightly high

- Not at all

5. To what extent do you think health care providers at ASTR prefer ordering medications with a PowerPlan versus without a PowerPlan?

- Extremely high

- Very high

- Moderately high

- Slightly high

- Not at all

6. To what extent do providers use PowerPlans when appropriate at ASTR?

- Extremely high

- Very high

- Moderately high

- Slightly high

- Not at all

7. How helpful are PowerPlans in reducing length of stay at ASTR?

- Extremely helpful

- Very helpful 
- Moderately helpful

- Slightly helpful

- Not at all

8. How helpful are PowerPlans in reducing mortality at ASTR?

- Extremely helpful

- Very helpful

- Moderately helpful

- Slightly helpful

- Not at all

9. At what degree do PowerPlans reduce pharmacy staff workload at ASTR?

- Extremely high

- Very high

- Moderately high

- Slightly high

- Not at all

10. Have you ever assisted in creating a PowerPlan?

- Yes

- No

11. If you responded "yes" or "maybe" to the previous question (question 10), please describe the PowerPlan that you helped to create. If you answered "no", please put N/A and move to the next question.

12. How likely would you work in a pharmacy setting that required creating and developing PowerPlans?

- Very likely

- Somewhat likely

- Somewhat unlikely

- Very unlikely

- Unsure

13. How likely would you be to recommend the use of PowerPlans in institutional and clinical settings throughout the United States?

- Very likely

- Somewhat likely

- Somewhat unlikely

- Very unlikely

- Unsure

\section{Demographics}

1. What is your age in years?

a. $20-25$ 

b. 26-29 years
c. 30-39 years
d. 40-49 years
e. $50+$ years

2. How many years have you worked as a pharmacist?
a. 0-2 years
b. 3-5 years
c. 6-10 years
d. 11-20 years
e. $20+$ years

3. How long have you worked at Ascension Saint Thomas Rutherford?
a. Less than 1 year
b. 1-2 years
c. 3-5 years
d. Greater than 5 years

4. What area of pharmacy do you practice?
a. Central pharmacy
b. Critical Care
c. Medical/Surgery
d. Other 


\section{Chapter 4}

\section{Results}

\section{Study Population}

The survey link allowed for responses for two weeks. There was a total of twenty-three pharmacists that responded to the survey. Each of them completed the entire survey. The summary of demographic findings was that the majority (56.5\%) of the participants were $30-39$ years of age. Eleven of the participants (47.8\%) practiced in medical/surgery area of ASTR. Six (26.1\%) of the participants practiced in the critical care area, and five (21.7\%) practiced in "Other." Other could include a range of areas such as administrative, staffing, etc. pharmacists. The majority (73.9\%) of the participants have worked as pharmacist for ten or less years. Figure 2 displays a chart of the participant time worked as pharmacists. Finally, the majority (52.2\%) of participants have worked specifically at ASTR for three years or more.

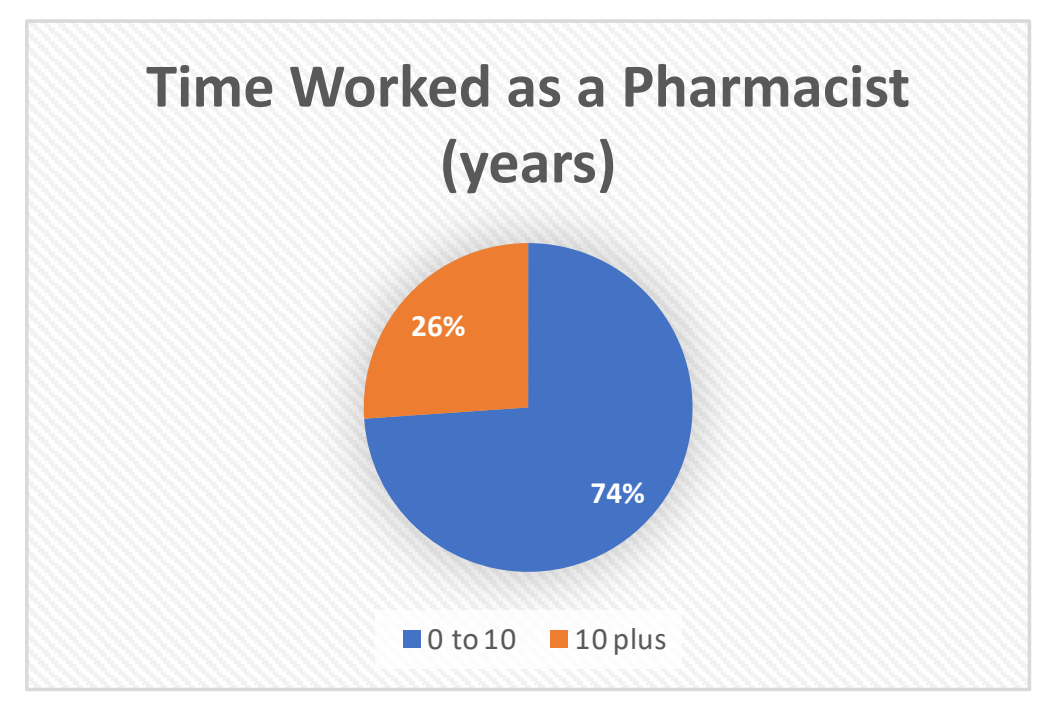

Figure 2 


\section{Perceptions of CPOEs}

$65.2 \%(15 / 23)$ of participants described their knowledge of CPOE and its use as extremely or very knowledgeable. The rest of the participants, $34.8 \%(8 / 23)$, described their knowledge of CPOE and its use as moderately knowledgeable. None of the participants described their knowledge of CPOE and its use as slightly knowledgeable or not at all.

$82.6 \%(19 / 23)$ of the participants thought that PowerPlans were very or extremely helpful in improving clinical decisions at ASTR. After combining two questions, it was determined that $21.7 \%$ (10/46) participants thought that PowerPlans were very helpful at reducing patient mortality and length of stay at ASTR. Though $47.8 \%(22 / 46)$ of participants thought that PowerPlans were moderately helpful at reducing patient mortality at ASTR. 13\% (3/23) thought that PowerPlans were not at all helpful at reducing patient length of stay at ASTR. 47.8\% (11/23) participants thought that PowerPlans had a moderately high extent at increasing microbial stewardship at ASTR. 52.2\% (12/23) of the participants have assisted in creating a PowerPlan. The descriptions of the PowerPlans that they have helped to create are included in Table 2.

\section{Table 2. Participant's Description of PowerPlan They Helped to Create}

$$
\begin{aligned}
& \text { Participant Description of PowerPlan Created } \\
& 1 \text { - Emergency department (ED) acute pain and stroke, misc. } \\
& 2 \text { - Retinopathy of prematurity (ROP) in neonates, NICU IV fluid sub phase } \\
& 3 \quad \text { - Multimodal pain } \\
& \text { - Acute ischemic stroke, intracranial hemorrhage (ICH) } \\
& 5 \text { - Insulin drip for hypertriglyceridemia } \\
& 6 \text { - ED envenomation's, addition of Anavip }{ }^{\circledR}
\end{aligned}
$$




\begin{tabular}{|c|c|}
\hline 7 & - Tenecteplase and stroke \\
\hline 8 & - Existing NICU PowerPlans, antibiotic dosing and frequency \\
\hline 9 & $\begin{array}{l}\text { - Infectious disease (ID) related such as pneumonia, daptomycin dosing, } C \text {. } \\
\text { difficile }\end{array}$ \\
\hline 10 & - Various pediatrics \\
\hline 11 & - Tenecteplase for safety, and calcitonin for appropriateness \\
\hline 12 & - Sotalol inititiation \\
\hline
\end{tabular}

$95.6 \%(22 / 23)$ of the participants were very or somewhat likely to recommend the use of PowerPlans in institutional and clinical settings throughout the United States. One participant (4.3\%) was unsure if they would recommend the use of PowerPlans in institutional and clinical settings throughout the United States. None of the participants were unlikely to recommend the use of PowerPlans in institutional and clinical settings throughout the United States. Not all responses are included in the results. A complete list of questions and responses is included in Appendix A. 


\section{Chapter 5}

\section{Discussion}

\section{Limitations of the Study}

One limitation to this research was that it was a single-center study. If it was multi-center, it would have provided a greater number of participants as well as increase the external validity. The clinical pharmacists at ASTR are primarily located in the emergency department, critical care units, and medical/surgery inpatient floors. However, clinical pharmacists' practice in a great deal more clinical settings in the US including ambulatory care, oncology, behavioral health, and pediatric areas. If this study was completed at a larger institution, it may have provided a larger variety of clinical pharmacists' roles and responses. Another limitation is the low representation of pharmacists greater than forty years old. The majority of participants were $30-39$ years of age and younger. While this is a limitation, the title of "clinical pharmacist" is a more recent role of a pharmacists and therefore would make the study population similar to real world clinical pharmacist ages. This study also did not use any major statistical analysis when gathering and reporting the results. It was not a major clinical study, but simply a singleauthor master's degree student research paper. If this study had more authors as well as more funding, results that were not solely descriptive could have been presented.

\section{Future Recommendations}

When brainstorming research questions for this study, the original plan was to study a medication safety measure before and after a CPOE was implemented into the EHR. However, due to the author not being a full-time employee within the study population's health care system, it was not going to be possible for her to look at patient charts within the EHR. Because of that limitation, it was necessary to complete research that did not involve the author using patient charts. Future studies could look at patient outcomes (i.g. medication safety, antimicrobial stewardship, length of stay, etc.) before a CPOE was implemented and compare these outcomes to after the CPOE was implemented. A 
study looking at those outcomes could possibly provide objective measures and show nominal data for how CPOEs impact patient care.

\section{Conclusion}

Results from this study were not meant to be robust findings that could prove statistical differences and change current practice. There were many obstacles that prevented the author to study objective measures within patient charts in the EHR. The goal of this study was to show the impact that HITECH has had on clinical pharmacy and the roles that clinical pharmacists' have by creating CPOEs. The goal of this study was reached and hopefully, this paper provides literature for clinical pharmacy and CPOEs. CPOEs were a meaningful use criteria measure by CMS. Eligible professionals could receive monetary incentives for meeting meaningful use criteria, yet pharmacists were not eligible professionals. This studied showed that clinical pharmacists are knowledgeable about CPOEs, they believe that CPOEs reduce medication errors, and they would recommend the use of CPOEs in clinical and institutional settings throughout the US. Finally, it showed that clinical pharmacists assist in creating CPOE templates and these CPOEs involve acute pain and stoke protocols, antimicrobial stewardship, and insulin drips. While pharmacists were not eligibly professionals by CMS, they have still been impacted by HITECH and have assisted in meeting meaningful use criteria by the use and development of CPOEs. 


\section{References}

Alfayez, O. M., Leal, S., \& Warholak, T. (2018). Documenting Stage 1 and 2 Meaningful Use criteria: A comparison of clinical pharmacists with other healthcare providers. American Journal of HealthSystem Pharmacy, 75, S24-S28. https://doi-org.ezproxy.uthsc.edu/10.2146/ajhp170150

American College of Clinical Pharmacy. (2020). About clinical pharmacists. ACCP. https://www.accp.com/about/clinicalpharmacists.aspx

Anderegg, S., \& Gumpper, K. (2012). What meaningful use means for pharmacy. American journal of health-system pharmacy: AJHP: official journal of the American Society of Health-System Pharmacists. 69. 890-4. 10.2146/ajhp110326.

Ascension. (2021). Visitor Information.

https://healthcare.ascension.org/locations/tennessee/tnnas/murfreesboro-ascension-saintthomas-rutherford/visitor-information

Center for Medicare and Medicaid Services. (2018). Stage 3 program requirements for providers attesting to their state's medicaid promoting interoperability (PI) programs. CMS.gov. https://www.cms.gov/Regulations-andGuidance/Legislation/EHRIncentivePrograms/Stage3Medicaid_Require Health Information Technology for Economic and Clinical Health Act of 2009. 13 U.S.C. § 13001 et seq. (2009).

https://www.healthit.gov/sites/default/files/hitech_act_excerpt_from_arra_with_index.pdf Mennemeyer, S. T., Menachemi, N., Rahurkar, S., \& Ford, E. W. (2016). Impact of the HITECH Act on physicians' adoption of electronic health records. Journal of the American Medical Informatics Association : JAMIA, 23(2), 375-379. https://doi.org/10.1093/jamia/ocv103 
Nelson, S. D., Poikonen, J., Reese, T., El Halta, D., \& Weir, C. (2017). The pharmacist and the EHR. Journal of the American Medical Informatics Association : JAMIA, 24(1), 193-197. https://doi.org/10.1093/jamia/ocw044

Office of the National Coordinator for Health Information Technology (ONC). (2018, March 21). What is computerized provider order entry? HealthIT.gov https://www.healthit.gov/faq/what-computerized-provider-orderentry\#: :text=Computerized\%20provider\%20order\%20entry\%20(CPOE,paper\%2C\%20fax\%2C\%20or\%20 telephone.

Prgomet, M., Li, L., Niazkhani, Z., Georgiou, A., \& Westbrook, J. I. (2017). Impact of commercial computerized provider order entry (CPOE) and clinical decision support systems (CDSSs) on medication errors, length of stay, and mortality in intensive care units: a systematic review and meta-analysis. Journal of the American Medical Informatics Association : JAMIA, 24(2), 413-422. https://doi.org/10.1093/jamia/ocw145

Washington, V., DeSalvo, K., Mostashari, F., \& Blumenthal, D. (2017). The HITECH Era and the Path Forward. The New England journal of medicine, 377(10), 904-906.

https://doi.org/10.1056/NEJMp1703370 


\section{Appendix A}

1. How do you describe your knowledge of computerized provider order entry (CPOE) and its use? 23 responses

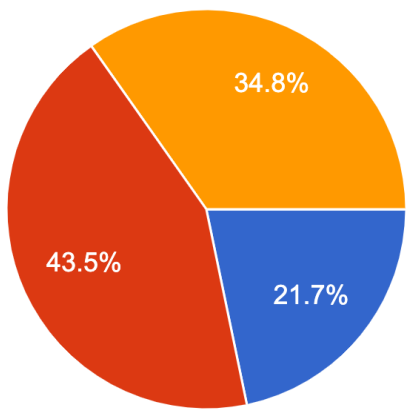

Extremely knowledgeable

Very knowledgeable

Moderately knowledgeable

Slightly knowledgeable

Not at all knowledgeable

2. How effective are PowerPlans in reducing medication errors at ASTR?

23 responses
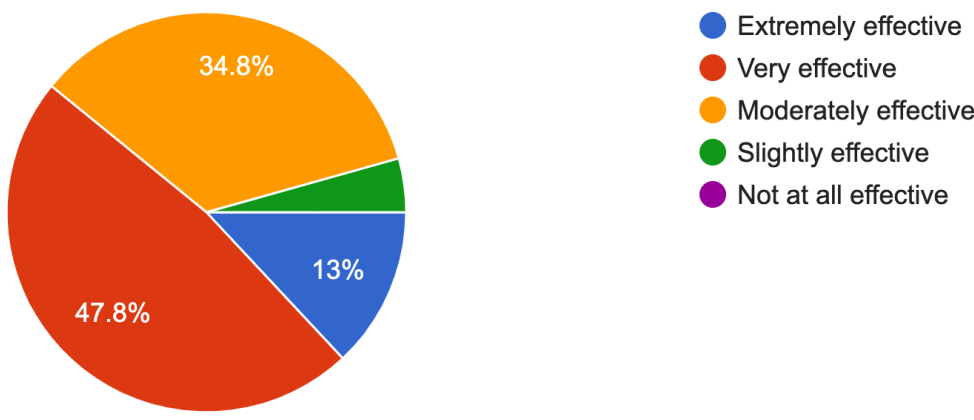

3. How helpful are PowerPlans to improve clinical decisions at ASTR?

23 responses

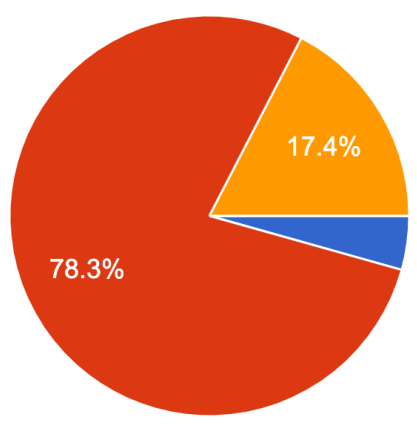

Extremely helpful

Very helpful

Moderately helpful

Slightly helpful

Not at all helpful 
4. To what extent do PowerPlans increase antimicrobial stewardship at ASTR? 23 responses

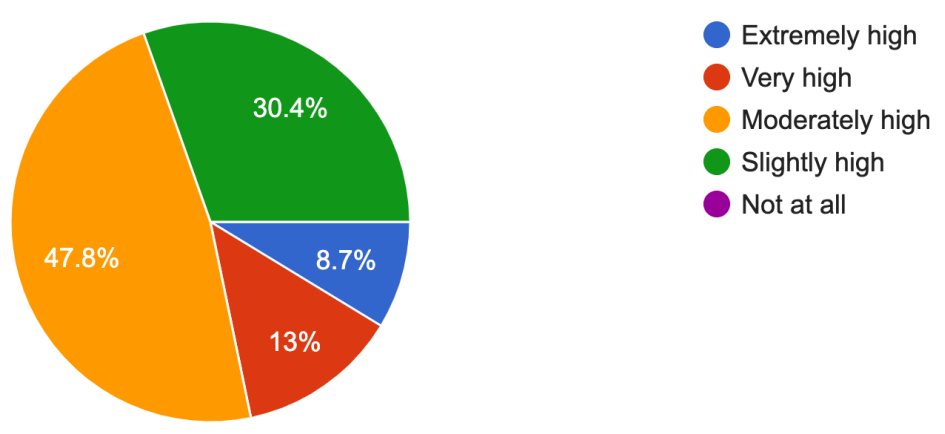

5. To what extent do you think health care providers at ASTR prefer ordering medications with a PowerPlan versus without a PowerPlan?

23 responses

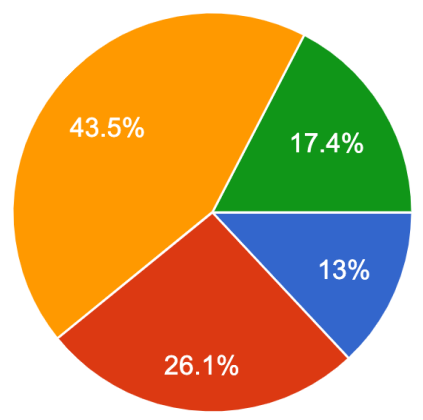

Extremely high

Very high

Moderately high

Slightly high

Not at all

6. To what extent do providers use PowerPlans when appropriate at ASTR? 23 responses

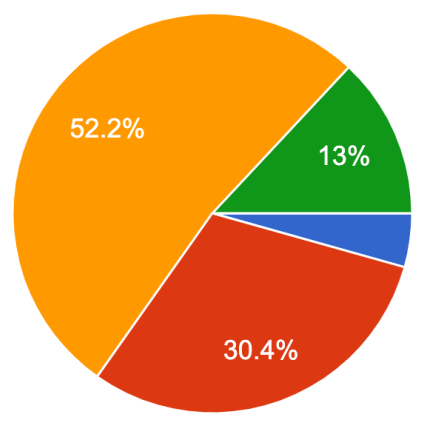
Extremely high
Very high
Moderately high
Slightly high
Not at all


7. How helpful are PowerPlans in reducing length of stay at ASTR? 23 responses

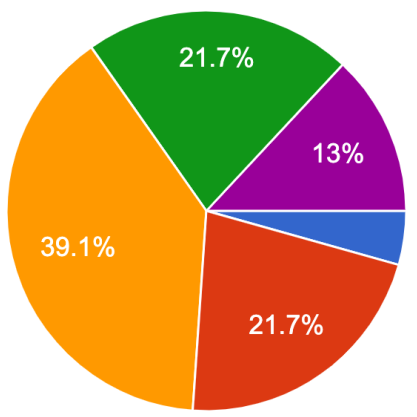

Extremely helpful

Very helpful

Moderately helpful

Slightly helpful

Not at all

8. How helpful are PowerPlans in reducing mortality at ASTR?

23 responses

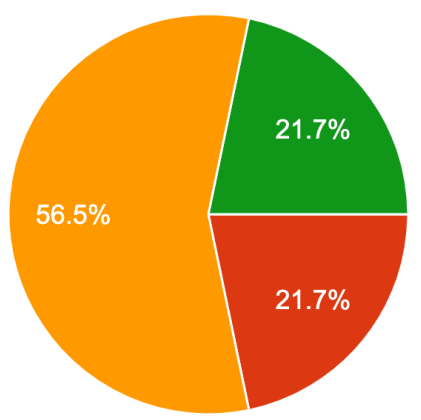

Extremely helpful

Very helpful

Moderately helpful

Slightly helpful

Not at all

9. At what degree do PowerPlans reduce pharmacy staff workload at ASTR? 23 responses

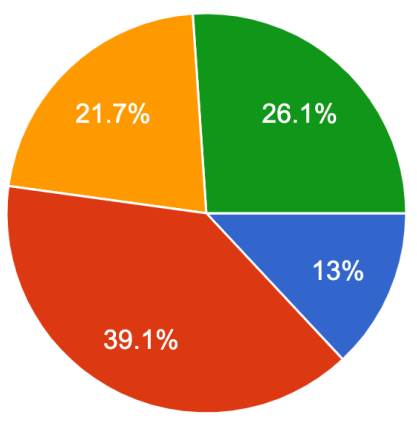

Extremely high

Very high

Moderately high

Slightly high

Not at all 
10. Have you ever assisted in creating a PowerPlan?

23 responses

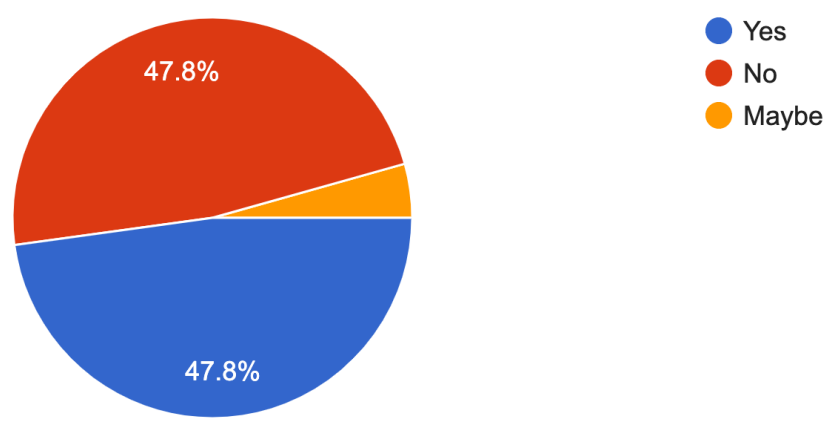

11. If you responded "yes" or "maybe" to the previous question (question 10), please describe the PowerPlan that you helped to create. If you answer...o", please put N/A and move to the next question. 19 responses

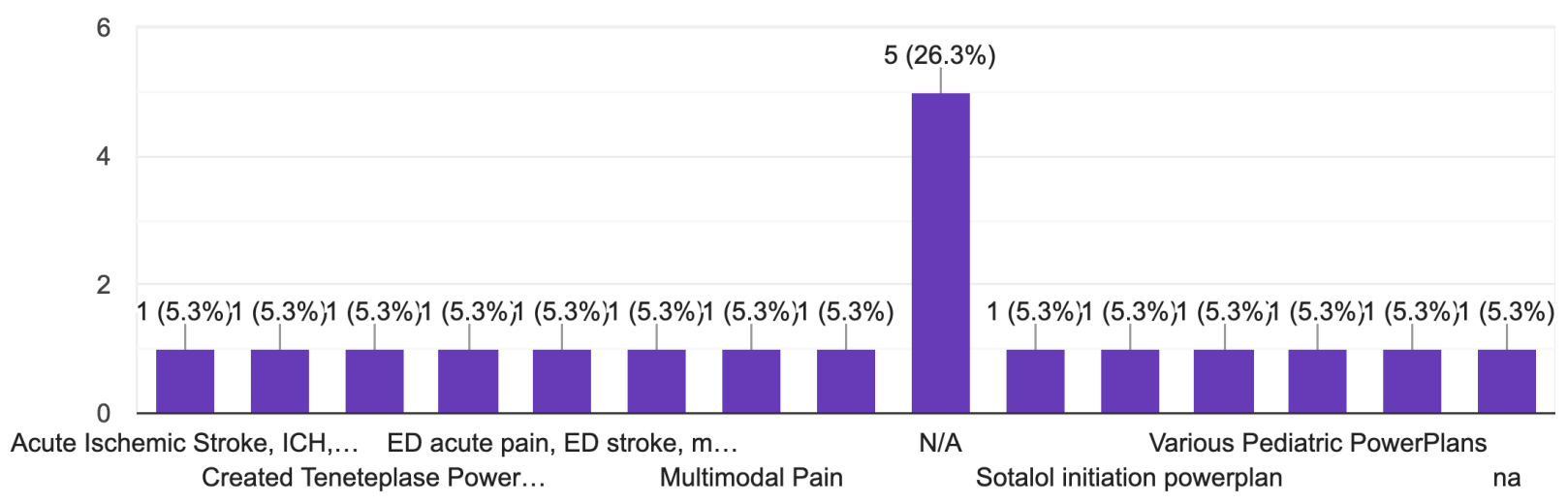

12. How likely would you work in a pharmacy setting that required creating and developing PowerPlans?

23 responses
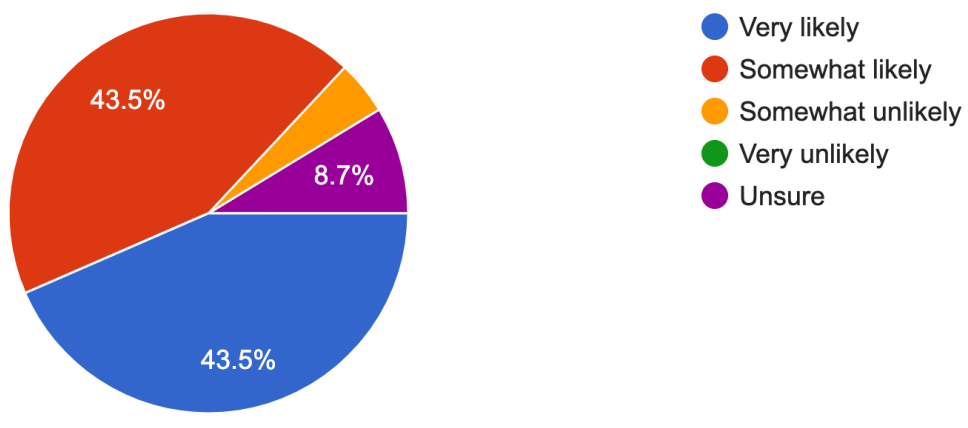
13. How likely would you be to recommend the use of PowerPlans in institutional and clinical settings throughout the Unites States?

23 responses

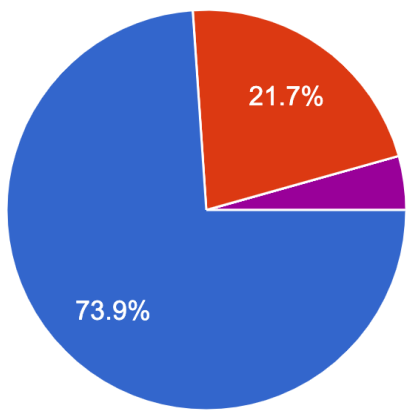

Very likely

Somewhat likely

Somewhat unlikely

Very unlikely

Unsure

\section{Demographics}

What is your age in years?

23 responses

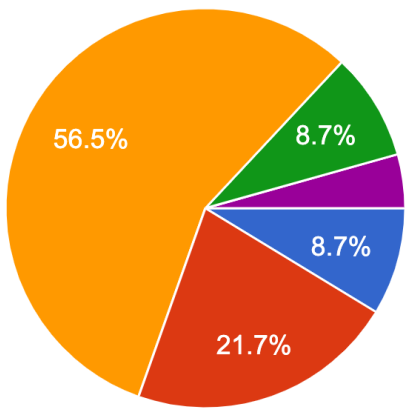

$20-25$

$26-29$

30-39

$40-49$

$50+$

How many years have you worked as a pharmacist? 23 responses

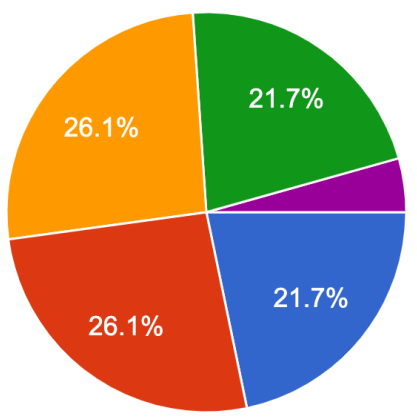


How long have you worked at Ascension Saint Thomas Rutherford?

23 responses

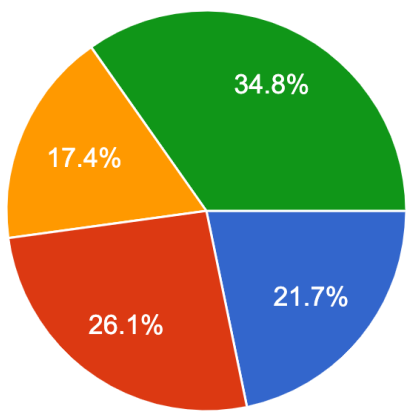

Less than 1 year

1-2 years

3-5 years

$5+$ years

What area of pharmacy do you practice?

23 responses

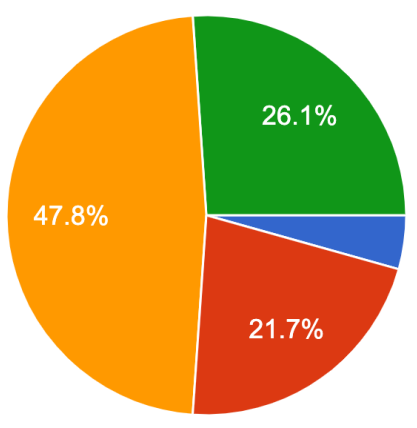

Central pharmacy

Critical Care

Medical/Surgery

Other 